\title{
Superconductor-insulator transition with a non-universal conductivity
}

\author{
Karl-Heinz Wagenblast ${ }^{a}$, Rosario Fazio $^{b}$, Gerd Schön $^{a}$, and Gergley Zimanyi ${ }^{c}$ \\ ${ }^{a}$ Institut für Theoretische Festkörperphysik, Universität Karlsruhe, 76128 Karlsruhe, Germany \\ ${ }^{b}$ Istituto di Fisica, Università di Catania, viale A. Doria, 95129 Catania, Italy \\ ${ }^{e}$ Department of Physics, University of California, Davis, CA 95616, USA
}

Motivated by experimental evidence for the importance of fermionic degrees of freedom near the superconductor-insulator transition, we take into account gapless electronic excitations for the description of the transition. Integrating them out yields a damping for the phase of the superconducting grains. We derive a Ginzburg-Landau-Wilson description for the onset of superfluidity which shows a non-ohmic dynamics. This dynamics depends qualitatively on the strength of the damping. Sufficiently strong damping modifies both, the critical behavior and the response near the transition. The critical exponents depend on the damping strength. The conductivity at the transition is finite but non-universal.

Films of superconducting material exhibit a superconductor-insulator transition [1]. A description in terms of boson models, where a well established gap is assumed also in the insulating phase, yields a universal value for the conductivity at the transition [2]. The experimental situation is richer. Especially in uniform films unpaired electrons seem to be important near the transition [1]. Therefore, as a first step for an extension of the boson models, we include gapless excitations into our approach. These yield a damping for the phase of the superconducting order-parameter. Our description differs from the model of Ref. [3], which takes into account continuous charge transfer between the different grains and yields a damping for the gradient of the phase. We take into account the coupling to unpaired electrons which yields a local damping for the phase. This has interesting consequences. The dynamics of a coarsegrained description for the transition depends qualitatively on the strength of the damping and modifies both, the critical behavior and the response at the transition. The concept of a universal response at the transition remains valid only for weak damping.

We use an effective action which, after integrating out the gapless excitations, reads

$$
\begin{aligned}
& S_{\text {eff }}[\varphi]=\frac{1}{2} \int_{0}^{\beta} \mathrm{d} \tau \mathrm{d} \tau^{\prime} \sum_{i} \alpha\left(\tau, \tau^{\prime}\right)\left[\varphi_{i}(\tau)-\varphi_{i}\left(\tau^{\prime}\right)\right]^{2} \\
& +\int_{0}^{\beta} \mathrm{d} \tau\left[\sum_{i} \frac{\dot{\varphi}_{i}(\tau)^{2}}{2 U}-J \sum_{\langle i j\rangle} \cos \left[\varphi_{i}(\tau)-\varphi_{j}(\tau)\right]\right] .(1)
\end{aligned}
$$

The first term describes the damping, which we choose to be ohmic, $\alpha\left|\omega_{\mu}\right| / 2 \pi$. The on-site charging energy $U$ and the Josephson coupling $J$ between neighboring islands are included. We decouple the Josephson coupling term by introducing the complex order-parameter field $\psi$ via a Hubbard-Stratonovich transformation. This yields the Ginzburg-LandauWilson (GLW) free energy functional $[4,5]$

$$
\begin{aligned}
& F=\int \mathrm{d}^{2} r \mathrm{~d} \tau \mathrm{d} \tau^{\prime} \bar{\psi}(r, \tau)\left\{\frac{1}{2 J}\left[1-\frac{\vec{\nabla}^{2}}{4}\right]\right. \\
& \left.-g\left(\tau-\tau^{\prime}\right)\right\} \psi\left(r, \tau^{\prime}\right)+\kappa \int \mathrm{d}^{2} r \mathrm{~d} \tau|\psi(r, \tau)|^{4}
\end{aligned}
$$

The dynamics of the field $\psi$ is governed by the phasephase correlator $g(\tau)=\left\langle\exp \left\{i \varphi_{i}(\tau)-i \varphi_{i}(0)\right\}\right\rangle_{0}$, which the presence of local damping decays algebraically in time $\left(\propto \tau^{-2 / \alpha}\right)$. The Fourier transform for small frequencies reads

$$
g\left(\omega_{\mu}\right)=g_{0}-\eta\left|\omega_{\mu}\right|^{s}-\zeta \omega_{\mu}^{2}, \quad \text { with } s=\frac{2}{\alpha}-1 .
$$

We notice that the free energy (2) contains a nonohmic dissipative term $\left(\alpha\left|\omega_{\mu}\right|^{s}\right)$ (reducing to ohmic at $\alpha=1$ ).

The transition occurs at the critical coupling which in the saddle point approximation is given by $J_{c r}=1 / 2 g_{0}$. Increasing damping shifts the phase boundary to smaller values of $J$. A quantum phase transition is ruled out beyond the critical value $\alpha=2$. The phase boundary for $T=0$ is shown in the 
inset of Fig. 1. The transition is of the 3D-XY class along the solid line, along the dotted line the critical exponents vary as a function of $\alpha$. Power counting arguments imply the dynamical critical exponent to be $z=\max (1,2 / s)$.

The conductivity can be expressed in terms of two and four point Green's functions [2]. In the Gaussian approximation the four point function factorizes. An analytic continuation $i \omega_{\mu} \rightarrow \omega+i \delta$ yields the realfrequency dependence $[5]$

$$
\begin{aligned}
& \sigma(\omega)=\frac{\sigma_{Q}}{2 \pi \omega} \int_{-\infty}^{\infty} \frac{\mathrm{d} x}{1-\mathrm{e}^{-\beta x}} \int_{0}^{\infty} \mathrm{d} k k^{3} \\
& {\left[G^{R}(k, x)-G^{A}(k, x)\right] \times\left[G^{R}(k, x)\right.} \\
& \left.+G^{A}(k, x)-G^{R}(k, x+\omega)-G^{A}(k, x-\omega)\right]
\end{aligned}
$$

$\sigma_{Q}=h / 4 e^{2}$. The advanced and retarded Greens functions are given by

$$
\begin{aligned}
& {\left[G^{A / R}(k, \omega)\right]^{-1}=\epsilon+k^{2}-\zeta \omega^{2}} \\
& +\eta|\omega|^{s}\left[\cos \frac{s \pi}{2} \pm i \operatorname{sign}(\omega) \sin \frac{s \pi}{2}\right],
\end{aligned}
$$

with $\epsilon=1 / 2 J-g\left(\omega_{\mu}=0\right)>0$. For low frequencies $\omega \ll \omega_{0}=\sqrt{4 \epsilon / \zeta}$, the real part of the conductivity is given by

$$
\operatorname{Re} \sigma(\omega)=\sigma_{Q} \frac{\eta^{2} \sin ^{2}\left(\frac{\pi}{2} s\right)}{6 \pi \epsilon^{2}} \frac{[,(1+s)]^{2}}{,(2+2 s)}|\omega|^{2 s}
$$

Of particular interest is the d.c. $(\omega \rightarrow 0)$ conductivity at the transition, i.e. for $\omega_{0} \rightarrow 0$ (with $\omega_{0} / \omega \rightarrow 0$ ). The value of the d.c. conductivity at the transition depends on the strength of the ohmic damping (for $\alpha>2 / 3$ ), as shown in Fig. 1 .

In conclusion we found that the local damping of the phase of the superconducting order-parameter yields a rich dynamics of a GLW description for the transition. This influences the critical behavior as well as the conductivity at the transition which we found to be non-universal for sufficiently strong damping. The non-ohmic dynamics in the GLW formulation leads to a vortex dynamics which reflects this dynamics, this will be reported in a forthcoming article. Our approach neglects the dynamics of the gap itself, which may be important for a vanishing gap. The studies show that new features occur near the superconductor-insulator transition in the presence of a local damping mechanism enabled by unpaired electrons.



Figure 1: Conductivity at the transition as a function of the damping strength $\alpha$. The inset shows the corresponding phase diagram. The conductivity at the transition is constant along the solid line, it varies along the dotted line. Along the dotted line also the critical exponents of the transition change.

\section{REFERENCES}

[1] H.M. Jaeger, D.B. Haviland, B.G. Orr, and A.M. Goldman, Phys. Rev. B 40, 182 (1989); D.B. Haviland, Y. Liu, and A.M. Goldman, Phys. Rev. Lett. 62, 2180 (1989); R.P. Barber, Jr. and R.C. Dynes, Phys. Rev. B 48, 10618 (1993); A. Yazdani and A. Kapitulnik, Phys. Rev. Lett. 74, 3037 (1995). S.-Y. Hsu, J.A. Chervenak, and J.M. Valles, Jr. Phys. Rev. Lett. 75, 132 (1995).

[2] M.P.A. Fisher, G. Grinstein, and S.M. Girvin, Phys. Rev. Lett. 64, 587 (1990); M.-C. Cha, M.P.A. Fisher, S.M. Girvin, M. Wallin, and A.P. Young, Phys. Rev. B 44, 6883 (1991).

[3] S. Chakravarty, G.-L. Ingold, S. Kivelson, and A. Luther, Phys. Rev. Lett. 56, 2303 (1986); S. Chakravarty, G.-L. Ingold, S. Kivelson, and G. Zimanyi, Phys. Rev. B 37, 3283 (1988).

[4] S. Doniach, Phys. Rev. B 24, 5063 (1981).

[5] A. van Otterlo, K.-H. Wagenblast, R. Fazio, and G. Schön, Phys. Rev. B 48, 3316 (1993). 